Korean elders: a population based study. International Psychogeriatrics, 30, 1-10.

Pellfolk, T., Gustadsson, T., Gustafson, Y. and Karlsson, S. (2009). Risk factors for falls among residents with dementia living in group dwellings. International Psychogeriatrics, 21, 187-194.

Stewart, I., Knight, C. and Johnson, C. (2008). Just how challenging can older people be? Part 2: Making the case for specialist services for risky and aggressive behaviour. PSIGE Newsletter, 103, 66-75.
Stubbs, B., Zapata, E. and Haw, C. (2009). Screening for osteoporosis: a survey of older psychiatric inpatients at a tertiary referral centre. International Psychogeriatrics, 21, 180-186.

\author{
Brendon Stubbs, Lead Physiotherapist \\ Townsend Division, St Andrews Healthcare, \\ Northampton, U.K. \\ Email: bstubbs@standrew.co.uk
}

\section{A replication of a cross-national study of the relationship between elderly suicide rates and urbanization}

Modernization is a social and economic process consisting of three interrelated processes of industrialization, urbanization and secularization. The process of industrialization may provide greater economic opportunities in urban areas and facilitate migration of people from rural to urban areas. This process of urbanization may lead to a weakening of ties with family, friends, local religious institutions and original place of residence. Difficulties in adjusting to the new urban environment may increase the risk of suicide and see a rise in suicide rates (Stack, 2000).

A cross-national study (Simpson and Concklin, 1989) and a within-country study (Stack, 1993) have both reported a positive correlation between urbanization and general population suicide rates. However, studies from advanced industrialized countries, including the U.S.A. and Japan, reported a negative correlation (Araki and Murata, 1986; Kowalski et al., 1987; Otsu et al., 2004). Moreover, a large cross-national study reported no such relationship (Zhang, 1998). Stack (1982; 2000) explained these conflicting findings by proposing a curvilinear (quadratic) relationship, whereby the suicide rate would initially increase in the early stages of urbanization due to social disruption following migration from rural to urban areas. Thereafter, the suicide rate would plateau, over several generations, as the new urban dwellers adjust to living in urban areas. This adjustment may ultimately lead to a gradual reduction in the suicide rate. Stack (2000) argued that this curvilinear relationship was supported by a longitudinal study of general population suicide rates in Finland (Stack, 1993). A recent crossnational study also reported this curvilinear (U- shaped curve) relationship between elderly suicide rates and urbanization only in males (Shah, 2008). The latter study used one-year cross-sectional data on elderly suicide rates and may be open to bias as suicide rates can randomly fluctuate year on year. Therefore, a study using one-year average of five years' data on elderly suicide rates and a more recent data set then used in the latter study was undertaken to replicate the earlier findings.

Data on elderly suicide rates for males and females in the age-bands 65-74 years and $75+$ years were ascertained from the World Health Organization (WHO) website (http://www. who.int/whosis/database/mort/table1.cfm). For a small number of countries only the raw figures for the number of suicides were available from the WHO website. Suicide rates for these countries were calculated by dividing the number of reported suicides by the population size in the relevant age-band and sex group available on the same website. Data were ascertained for the latest five consecutive years. The one-year average suicide rate was calculated by dividing the sum of suicide rates for the latest five consecutive years by five. The median (range) for the latest year for the suicide rate data was 2005 (1983-2007). Urbanization, in accordance with standard practice in suicide research, was defined as the percentage of the population living in urban areas (Simpson and Concklin, 1989). Data on the percentage of population living in urban areas was also ascertained from the WHO website (http://www.who.int/countries/afg/en/) and were for the year 2006 .

Curve estimation regression models were used to examine the curvilinear relationship between elderly suicide rates and the percentage of the population living in urban areas fitting the quadratic equation $y=a+b x-c x^{2}$ (where $y$ is the suicide rate, $x$ is the percentage of the population living in urban areas and $a, b$, and $c$ are constants).

A full data set for elderly suicide rates and the percentage of the population living in urban areas was available for 85 countries. Table 1 illustrates 
Table 1. Curve estimation regression models for the relationship between elderly suicides and the percentage of the population living in urban areas

\begin{tabular}{llllll}
\hline & & DEGREES OF & & \\
& $\mathrm{R}^{2}$ & FREEDOM & F VALUE & SIGNIFICANCE & REGRESSION EQUATION \\
Males 65-74 years & 0.05 & 82 & 2.20 & $\mathrm{NS}$ & $y=-16.26+1.43 x-0.01 x^{2}$ \\
Males 75+ years & 0.08 & 82 & 3.33 & $\mathrm{P}=0.041$ & $y=-36.26+2.28 x-0.02 x^{2}$ \\
Females 65-74 years & 0.09 & 82 & 4.04 & $\mathrm{P}=0.021$ & $y=-7.98+0.43 x-0.003 x^{2}$ \\
Females 75+ years & 0.03 & 82 & 1.12 & $\mathrm{NS}$ & $y=-4.67+0.42 x-0.003 x^{2}$ \\
\hline
\end{tabular}

$y=$ suicide rates.

$x=$ percentage of people living in urban areas.

$a, b$ and $c$ are constants.

NS $=$ not significant.

the characteristics of the curve estimation regression models for males and females in both the elderly age-bands. There was a statistically significant relationship between male suicide rates in the ageband 75+ years and females in the age-band 6574 years and the percentage of the population living in urban areas was curvilinear (U-shaped curve) and fitted the quadratic equation $y=a+b x-c x^{2}$. There was an absence of a significant such curvilinear relationship for males in the age-band 65-74 years and females in the age-band $75+$ years.

This study did not confirm the exact findings of the earlier similar study, which had used oneyear cross-sectional data on elderly suicide rates (Shah, 2008). Moreover, the absence of consistency in the findings from the current study, using oneyear average of five years data on suicide rates and a more recent data set than the earlier study, suggests that the observed curvilinear relationship between elderly suicide rates and urbanization may be spurious. This may also explain the conflicting findings in the literature summarized above. Many of the well-described methodological difficulties in using cross-national data on elderly suicide rates (Shah, 2008) may, in part, explain the lack of consistency in the findings. Within-country longitudinal studies are required to clarify this controversial curvilinear relationship.

\section{Conflict of interest}

None.

\section{References}

Araki, S. and Murata, K. (1986). Social life factors affecting suicide in Japanese men and women. Suicide and Life Threatening Behaviour, 16, 458-468.

Kowalski, G. S., Faupel, C. E. and Starr, P. D. (1987). Urbanization and suicides: a study of American counties. Social Forces, 66, 85-101.

Otsu, A., Araki, S., Sakai, R., Yokoyama, K. amd Voorhees, A. S. (2004). Effects of urbanization, economic development, migration of workers on suicide mortality in Japan. Social Sciences and Medicine, 58, 1137-1146.

Shah, A. K. (2008). A cross-national study of the relationship between elderly suicide rates and urbanization. Suicide and Life Threatening Behaviour, 38, 714-719.

Simpson, M. and Concklin, G. (1989). Socio-economic development, suicide and religion: a test of Durkhiem's theory of religion and suicide. Social Forces, 67, 945964.

Stack, S. (1982). Suicide: a decade review of sociological literature. Deviant Behavior, 4, 41-66.

Stack, S. (1993). The effect of modernization on suicide in Finland, 1800-1985. Sociological Perspective, 36, 137-148.

Stack, S. (2000). Suicide: a 15-year review of the sociological literature. Part II: Modernization and social integration perspectives. Suicide and Life Threatening Behaviour, 30, 163-176.

Zhang, J. (1998). Suicide in the world: toward a population increase theory of suicide. Death Studies, 22, 525-539.

\section{AJiT SHaH}

Professor of Ageing, Ethnicity and Mental Health, University of Central Lancashire, Preston, U.K. and Consultant Psychiatrist, West London Mental Health NHS Trust, London, U.K.

Email: ajit.shah@wlmht.nhs.uk 